\title{
Observations of in-situ generated gravity waves during a stratospheric temperature enhancement (STE) event
}

\author{
A. J. Gerrard ${ }^{1}$, Y. Bhattacharya ${ }^{1}$, and J. P. Thayer ${ }^{2}$ \\ ${ }^{1}$ Center for Solar-Terrestrial Research, New Jersey Institute of Technology, 323 Martin Luther King Jr. Boulevard, Newark, \\ NJ 07102-1982, USA \\ ${ }^{2}$ Aerospace Engineering Sciences, University of Colorado, 429 UCB, Boulder, CO 80309-0429, USA
}

Received: 21 February 2011 - Published in Atmos. Chem. Phys. Discuss.: 9 May 2011

Revised: 25 October 2011 - Accepted: 11 November 2011 - Published: 30 November 2011

\begin{abstract}
Evidence for in situ generated atmospheric gravity waves associated with a stratospheric temperature enhancement (STE) are presented. The signatures of two sets of gravity waves are observed by molecular-aerosol lidar in conjunction with the early December 2000 STE event above Sondrestrom, Greenland. The first set of gravity waves shows downward phase progression with a vertical wavelength of $\sim 8 \mathrm{~km}$ while the second set shows upward phase progression with a vertical wavelength of $\sim 9 \mathrm{~km}$. With estimates of the background wind fields from synoptic analyses, the various intrinsic gravity wave parameters of these two wave structures are found. The observed wave features compare well to previous numerical modeling predictions.
\end{abstract}

\section{Introduction}

Stratospheric temperature enhancements (STEs) have been experimentally observed, theoretically and numerically modeled, and discussed in the scientific literature for some time, often under differing nomenclature such as: stratospheric baroclinic zones, stratospheric sudden warmings (SSWs), winter stratospheric temperature anomalies, etc. SSW or a major warming is defined by the World Meteorological Organization as an increase in zonal-mean temperature of the polar region such that the net zonal-mean zonal winds become easterly north of $60^{\circ} \mathrm{N}$ at $10 \mathrm{hPa}$ or below. An STE, such as the one discussed in this paper, leaves the lower stratosphere relatively undisturbed from a global context and is therefore

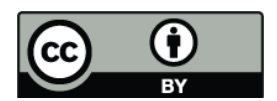

Correspondence to: A. Gerrard (gerrard@njit.edu) considered distinct from an SSW. Meriwether and Gerrard (2004) have reviewed the phenomenology of STEs and have highlighted the interesting attributes of the structures, including (1) the nature of their development and evolution, (2) how STEs may serve as precursors to sudden stratospheric warmings, and (3) the role of STEs as in situ generators of middle atmospheric gravity waves. The first issue has been addressed by a number of authors, including a companion paper by Thayer and Livingston (2008). This paper addresses the third issue by analyzing a STE event in December 2000, when the height of the stratopause dropped to $\sim 42 \mathrm{~km}$ and temperatures were enhanced by $\sim 50 \mathrm{~K}$, described in that earlier Thayer and Livingston (2008) paper.

As noted in the review by Meriwether and Gerrard (2004), numerical modeling of STE events by Fairlie et al. (1990) suggest that gravity waves are generated at the location of the STE. More recently, Vadas and Fritts (2001), and Fritts et al. (2006) have discussed strong temperature anomalies and subsequent local adjustment as potential generators of gravity waves. As reproduced from Fairlie et al. (1990) in Fig. 1, upward propagating gravity waves are seen in model runs with horizontal wavelengths of $\sim 1200 \mathrm{~km}$, vertical wavelengths of $\sim 8 \mathrm{~km}$, and intrinsic periods of $\sim 10 \mathrm{~h}$. Though single large-scale wave such as this does not transport large amounts of horizontal momentum vertically, their presence may nonetheless assist smaller-scale gravity waves to move towards convective or dynamical instability due to localized wave-wave interactions (Fritts and Alexander, 2003). Furthermore, it is not clear to what extent the associated localized momentum deposition plays a role in the re-stabilization of the high latitude middle atmosphere system (i.e., the reformation of the polar vortex) and return to geostrophy. 


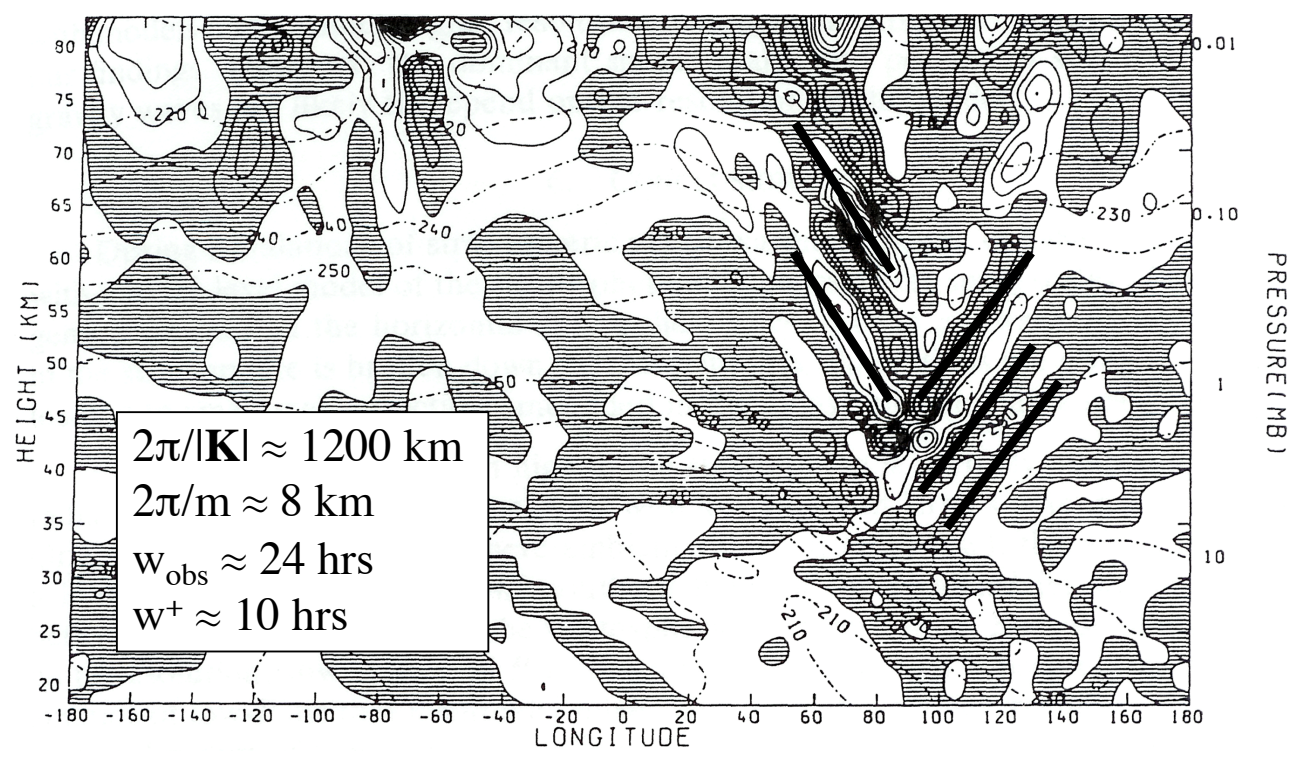

Fig. 1. From Fairlie et al. (1990). Simulated cross-section of temperature (dashed, units of K) and the divergence of the horizontal wind (solid, units of $10^{-6} \mathrm{~s}^{-1}$ ) at $57.5^{\circ} \mathrm{N}$ on 25 February 1979. Regions of convergence are shaded. Note that the the original pressure axis of millibars (written as $\mathrm{mB}$ ) has been retained. The figure has been modified to highlight modeled, upwardly propagating gravity waves to both the west and east of the STE/baroclinic zone. Estimated gravity wave parameters are listed.

In situ observations of STE-associated gravity waves have not been reported (Meriwether and Gerrard, 2004). To our knowledge, this paper presents the first observations of such gravity wave signatures and associated wave structures generated during an STE event. The observations were taken during 11-12 December 2000 above Sondrestrom, Greenland. Synoptic scale conditions prevalent during this STE were presented in Thayer and Livingston (2008); this study deals exclusively with the period of peak STE temperatures over Sondrestrom. In Sect. 2 we present a brief overview of the instrumentation and analysis, in Sect. 3 we discuss the gravity wave characteristics, and in Sect. 4 we discuss these findings.

\section{Instrumentation and analysis}

Data used in this study originate from the ARCLITE molecular-aerosol lidar system located at Sondrestrom, Greenland. The ARCLITE system is discussed in detail in Thayer et al. (1997) and data from this system consist of range-time resolved temperature profiles of the upper stratosphere and lower mesosphere, relative gravity wave activity in the upper stratosphere, and aerosol volume backscatter coefficients. The data products have been used to explore mesospheric clouds (Thayer et al., 2003), relationship between gravity waves and mesospheric clouds (Gerrard et al., 1998, 2004a,b), thermal and gravity wave climatologies (Gerrard et al., 2000), and the synoptic development of the polar vortex (Gerrard et al., 2002). In Fig. 2 we present interpolated temperature profiles taken by the Sondrestrom lidar system in early December 2000 (Thayer and Livingston, 2008). Measurements were taken on the days indicated by black markers and interpolated across the entire duration of the STE event. Uncertainty of the lidar-deduced temperatures is less than $3 \mathrm{~K}$ below $70 \mathrm{~km}$. Of particular note is the lidar data from 11-12 December [day of year 346-347] , whose time evolution is also portrayed in Fig. 3. The temperature data here was low pass filtered in the vertical with a cutoff at $7 \mathrm{~km}$. Uncertainty on the measured temperatures is less than $5 \mathrm{~K}$ below $74 \mathrm{~km}$. In these data the STE is clearly evident as a narrow ridge of temperatures exceeding $320 \mathrm{~K}$; over $40 \mathrm{~K}$ above the climatological value (Gerrard et al., 2000).

From the $\sim 25 \mathrm{~h}$ long set of molecular - aerosol lidar data spanning this time period, it is possible to obtain timeresolved relative atmospheric density perturbations between $30 \mathrm{~km}$ and $70 \mathrm{~km}$ as outlined in Gerrard et al. (1998, 2004b). The density perturbation data (relative to an estimated atmospheric background), available in $5 \mathrm{~min}$ by $192 \mathrm{~m}$ vertical resolution bins, have been low pass filtered with cutoffs at $2 \mathrm{~km}$ in the vertical and $30 \mathrm{~min}$ in the temporal. These relative density perturbations are shown in Fig. 3 .

In a perturbation analysis of the base-state parameters of an ideal gas, it can be shown that regions of high density perturbations correspond to regions of lower temperatures. This is the case on inspection of Figs. 2 and 3, where high density perturbations are $180^{\circ}$ out of phase with the corresponding temperatures. As such, Figs. 2 and 3 essentially show the same thing, but gravity wave signatures are more readily extracted and analyzed in the relative density perturbations. From these figures, one sees that the STE peak temperatures 


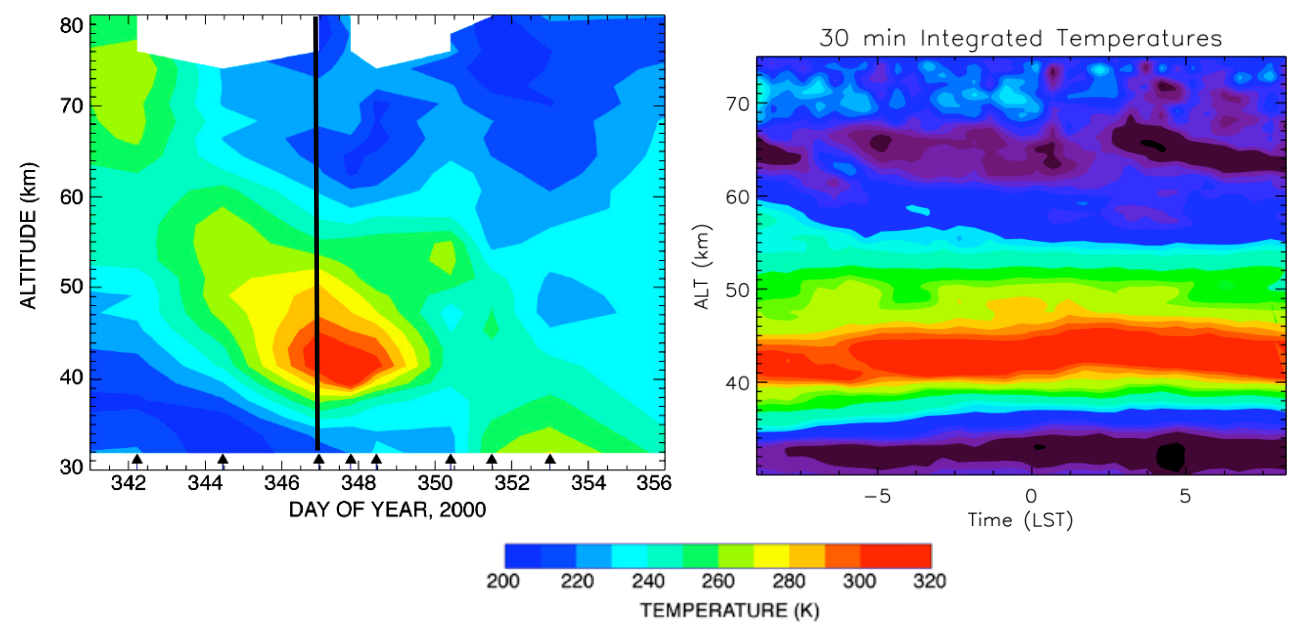

Fig. 2. From Thayer and Livingston (2008). Evolution of middle atmospheric temperatures over Sondrestrom, Greenland in December 2000 as measured by the molecular/aerosol lidar system. The vertical black line represents the day under study herein. (right) Range-time temperature evolution over Sondrestrom over the day near the estimated peak STE represented by the vertical black line (i.e., day of year 346-347, night of 11-12 December 2000, leap year). Time is local solar time (LST) with 00:00 representing midnight of 11 December.

at $\sim 42 \mathrm{~km}$ fluctuates by a few kilometers in height and in peak intensity through the course of the observations. This is presumably due to the time-varying nature of the baroclinic zone and other atmospheric structures, like gravity wave activity.

\section{Gravity wave structures}

Inspection of Fig. 3 shows at least two regions of relatively coherent wave-phase progression. These two areas of wavelike structure are highlighted in Fig. 3, where the waves in the upper left corner (centered at $\sim 04: 00 \mathrm{LST}$ and $\sim 65 \mathrm{~km}$, and indicated by red streaks sketched by inspection of the data) are referred to as "set 1", and the waves on the right (centered at $\sim 09: 00] \mathrm{LST}$ and $\sim 50 \mathrm{~km}$, and represented by blue streaks) are referred to as "set 2." No wave structures were apparent in the data below $\sim 45 \mathrm{~km}$.

Addressing wave set 1 , the wave structure has a downward phase progression in time, a vertical wavelength of $\sim 8 \mathrm{~km}$ and an observed period of $\sim 5 \mathrm{~h}$. This type of structure is common over Sondrestrom (for e.g., seen in data presented in Gerrard et al., 1998 and Gerrard et al., 2000), and has the characteristic of an atmospheric gravity wave propagating upwards in height (i.e., the phase progression is downward, which is indicative of upward energy progression). Strictly speaking, downward phase propagation results in upward propagation of energy in a fluid at rest or in a frame moving with the background wind. This would hold approximately true if the polar vortex mass did not move significantly during the STE event. Such an assumption is not unreasonable; for instance, analysis presented in Bhattacharya and Gerrard (2010) using stratospheric assimilated data from

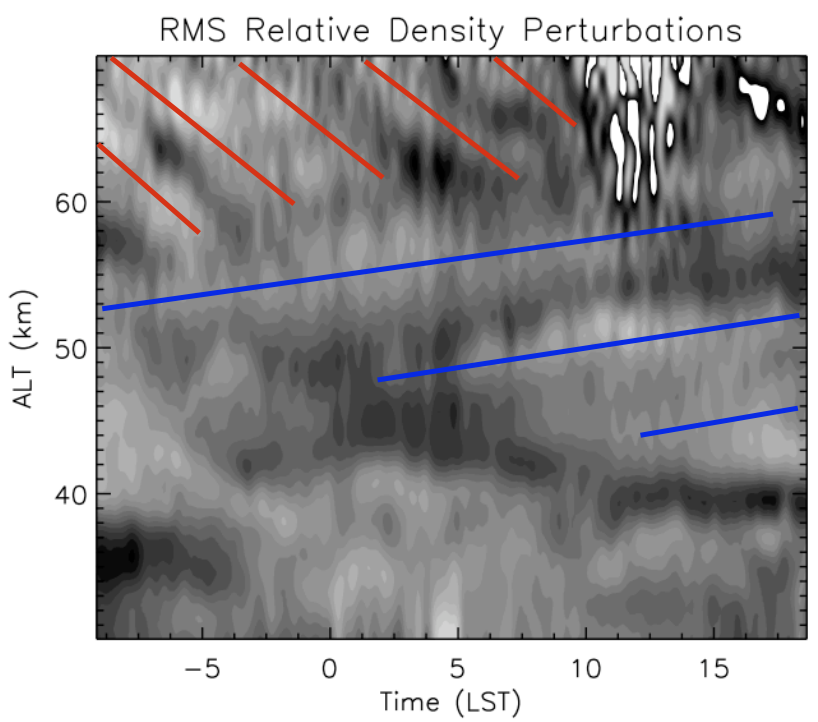

Fig. 3. Relative upper stratospheric and lower mesospheric density perturbations observed by the molecular/aerosol lidar system at Sondrestrom for the same range-time of Fig. 2. The relative density perturbations are scaled from $-10 \%$ to $10 \%$ in 20 grey-scale intervals. Black represents the negative perturbations, while white represents the positive perturbations. Solar noise is present in the 60- to $70 \mathrm{~km}$ region of the figures around 12:00 LST which prohibited robust calculation of the temperatures in Fig. 2. Phase peaks of upward and downward phase lines corresponding to the two gravity wave sets are determined by inspection and highlighted.

United Kingdom Met Office (UKMO) shows that geopotential height at $10 \mathrm{hPa}$ remained relatively constant $(\sim 36 \mathrm{~km})$ over Resolute Bay $\left(75^{\circ} \mathrm{N}, 95^{\circ} \mathrm{W}\right)$ during the STE period 
reported here. Assimilated data from National Center for Environmental Prediction (NCEP) gives the average horizontal wind velocity $\sim 50 \mathrm{~m} \mathrm{~s}^{-1}$ at $\sim 65 \mathrm{~km}$.

If the wind velocity vector $\boldsymbol{U}$ is aligned with the gravity wave horizontal wavenumber vector $\boldsymbol{U}$ (in general, gravity waves with such aspect ratios propagate in the direction of wind as modeled in Gerrard et al., 2004a), and using the buoyancy frequency $N$ deduced from the nightly averaged temperature profile, the horizontal wavelength and intrinsic period $\omega^{+}$of the wave can be estimated from the gravity wave dispersion relationship in the form:

$\left(\omega^{+}\right)^{2}=(\omega-\boldsymbol{U} \boldsymbol{k})^{2}=\frac{N^{2} k^{2}+f^{2} m^{2}}{k^{2}+m^{2}}$

where, aside from the variables already described earlier, $\omega$ is the ground-based frequency, $f$ is the Coriolis parameter, and $m$ is the gravity wave vertical wavenumber. The group velocity of the gravity wave can also be determined from this equation by taking the derivative of $w$ with respect to the horizontal or vertical wavenumber to yield the horizontal group velocity or vertical group velocity, respectively.

Numerical solution of the dispersion relation using a Laguerre method yields two real roots and two imaginary roots. The imaginary roots are discarded as they represent evanescent structures. The first real root results in a gravity wave with a horizontal wavelength of $\sim 1700 \mathrm{~km}$ and an intrinsic wave period of $\sim 10.5 \mathrm{~h}$, with positive horizontal and vertical group velocities indicating an upward propagating gravity wave moving in the direction of the background wind. The second root yields horizontal wavelength of $\sim 400 \mathrm{~km}$ and an intrinsic wave period of $\sim 4 \mathrm{~h}$, with a positive horizontal group velocity and negative vertical group velocity indicating a downward propagating gravity wave moving in the direction of the background wind.

Addressing wave set 2, the wave structure has an upward phase progression in time, a vertical wavelength of $\sim 9 \mathrm{~km}$, and an observed period of $\sim 26 \mathrm{~h}$. Estimating that the background wind velocity from NCEP analyses to be $+70 \mathrm{~m} \mathrm{~s}^{-1}$, coupled with the assumption of wave vector $k$ approximately aligned with $U$, the first real root yields a gravity wave with a horizontal wavelength of $\sim 1870 \mathrm{~km}$ and an intrinsic wave period of $\sim 10.4 \mathrm{~h}$, with positive horizontal and vertical group velocities indicating an upwards propagating gravity wave moving in the direction of the background wind. The second real root yields a wave with a horizontal wavelength of $\sim 6200 \mathrm{~km}$ and an intrinsic wave period of $\sim 12.6 \mathrm{~h}$, with a positive horizontal group velocity and negative vertical group velocity indicating a downward propagating gravity wave moving in the direction of the background wind.

It can be shown that uncertainties of $\pm 1 \mathrm{~km}$ in the vertical wavelengths, $\pm 1 \mathrm{~h}$ in the observed periods, and $\pm 10 \mathrm{~m} \mathrm{~s}^{-1}$ do not dramatically alter the estimated horizontal wavelengths and intrinsic periods.

\section{Conclusions}

Assuming that the first real root of set 1 and the first real root of set 2 are the physically meaningful roots (i.e., both indicating upwards gravity waves propagating from the STE region which fits the physical model), the observed gravity wave characteristics compare very well with model output of Fairlie et al. (1990). Furthermore, there was no indication of any wave structures below the STE temperature peak (i.e., between $30 \mathrm{~km}$ and $45 \mathrm{~km}$ ) to indicate a gravity wave passing through the STE. As such, we conclude that this is strong evidence for an observation of upward propagating gravity waves generated in situ by a STE event.

It should be noted that higher than normal total ozone content were reported in the Arctic vortex (until $63^{\circ} \mathrm{N}$ latitude for which ozone data was available) for the 20002001 winter; for December 2000, total ozone was higher by $\sim 20, \%$ compared to early 1980s (NCEP-NOAA Winter Bulletin, 2000-2001). An unusual and extensive $\sim 3 \mathrm{~km}$ thick aerosol cloud layer, spanning almost the entire polar vortex around $\sim 38 \mathrm{~km}$ altitude also formed in the first two weeks of December 2000, as reported by Gerding et al. (2003) observed from four different Arctic stations. Bhattacharya et al. (2004) have also reported increased gravity wave activity in the mesopause (hydroxyl airglow layer) polar vortex immediately following the STE events described in this paper. These observations in the current context increase the likelihood that the temperature enhancement, and the associated GW generation had their origins within a relatively shallow layer in the stratosphere. The long wavelength observed is also suggestive of a horizontally extensive forcing and a slower adjustment process (Fritts et al., 2006), unlike a vertically extended source typically found around the edges of a split vortex.

Based on this one observation, we are currently searching through the NCEP geopotential data archive for similar polar vortex configurations seen during this event. However, the combination of observing conditions, namely (a) the disruption of the polar vortex over Sondrestrom and actual occurrence of an STE when (b) there are clear sky conditions in which (c) the ARCLITE lidar system was collecting data is quite rare. Utilization of other lidar data archives, e.g., from the ALOMAR lidar system, the Poker Flat Lidar system, and the Eureka lidar system, would likely increase the probability of observing gravity waves associated with a STE.

In conclusion, in situ forcing of middle atmospheric gravity waves are of interest to the upper and lower atmospheric communities because, to date, little is known about the contribution that such waves may play in the global circulation. In this study we have demonstrated the likely origin of such in situ forcing associated with an observed STE. Further studies are necessary to better understand the role of such waves in the high latitude environment, how frequently such waves are generated, and the synoptic impacts on the surrounding atmospheric environment. 
Acknowledgements. The authors of this paper would like to thank site members of the Sondrestrom research facility and J. Livingston (SRI) for their support. AG and YB were supported in part by the National Science Foundation through ATM-0735452 and NSF-ATM-0457277.

Edited by: F.-J. Lübken

\section{References}

Bhattacharya, Y. and Gerrard, A. J.: Wintertime mesopause region vertical winds from Resolute Bay, J. Geophys. Res, 115, D00N07, doi:10.1029/2010JD014113, 2010.

Bhattacharya, Y., Shepherd, G. G., and Brown, S.: Variability of atmospheric winds and waves in the Arctic polar mesosphere during a stratospheric sudden warming, Geophys. Res. Lett., 31, L23101, doi:10.1029/2004GL020389, 2004.

Fairlie, T. D. A., Fisher, M. and O'Neill, A.: The development of narrow baroclinic zones and other small-scale structure during simulated major warmings, Q. J. Roy. Meteorol. Soc., 116, 287$315,1990$.

Fritts, D. C. and Alexander M. J.: Gravity wave dynamics and effects in the middle atmosphere, Rev. Geophys., 41, 1003, doi:10.1029/2001RG000106, 2003.

Fritts, D. C., Vadas, S. L., Wan, K., and Werne, J. A.: Mean and variable forcing of the middle atmosphere by gravity waves, J. Atmos. Sol.-Terr. Phys., 68, 247-265, 2006.

Gerding, M., Baumgarten, G., Blum, U., Thayer, J. P., Fricke, K.H., Neuber, R., and Fiedler, J.: Observation of an unusual midstratospheric aerosol layer in the Arctic: possible sources and implications for polar vortex dynamics, Ann. Geophys., 21, 10571069, doi:10.5194/angeo-21-1057-2003, 2003.

Gerrard, A. J., Kane, T. J., and Thayer, J. P: Noctilucent clouds and wave dynamics: Observations at Sondrestrom, Greenland, Geophys. Res. Lett., 25, 2817-2820, 1998.
Gerrard, A. J., Kane, T. J., and Thayer, J. P.: Year-round temperature and wave measurements of the arctic middle atmosphere for 1995-1998, in Geophysical Monograph Series 123: Atmospheric Science Across the Stratopause, edited by D. E. Siskind, S. D. Eckermann, and M. E. Summers, American Geophysical Union, Washington, DC, USA, 83, 488, 2000.

Gerrard, A. J., Kane, T. J., Thayer, J. P., Duck, T. J., Whiteway, J. A., and Fiedler, J.: Synoptic-scale study of the arctic polar vortex's influence on the middle atmosphere: I. observations, J Geophys. Res., 107, 4276, doi:10.1029/2001JD000681, 2002.

Gerrard, A. J., Kane, T. J., Eckermann, S. D, and Thayer, J. P.: Gravity waves and mesospheric clouds in the summer middle atmosphere: A comparison of lidar measurements and ray modeling of gravity waves over Sondrestrom, Greenland, J. Geophys. Res., 109, D10103, doi:10.1029/2002JD002783, 2004a.

Gerrard, A. J., Kane, T. J., Thayer, J. P., and Eckermann, S. D.: Concerning the upper stratospheric gravity wave and mesospheric cloud relationship over Sondrestrom, Greenland, J. Atmos. Sol.Terr. Phys., 66, 229-240, 2004b.

Meriwether, J. W. and Gerrard, A: Mesosphere inversion layers and stratosphere temperature enhancements, Rev. Geophys., 42, RG3003, doi:10.1029/2003RG000133, 2004.

Thayer, J. and Livingston, J: Observations of wintertime arctic mesospheric cooling associated with stratospheric baroclinic zones, Geophys. Res. Lett., 35, L10109, doi:10.1029/2008GL034955, 2008.

Thayer, J. P., Nielsen, N. B., Warren, R., Heinselman, C. J., and Sohn, J.: Rayleigh lidar system for middle atmosphere research in the arctic, Opt. Eng., 36, 2045-2061, 1997.

Thayer, J. P., Rapp, M., Gerrard, A. J., Gudmundsson, E., and Kane, T. J.: Gravity-wave influences on arctic mesospheric clouds as determined by a rayleigh lidar at Sondrestrom, Greenland, J. Geophys. Res., 108, 8449, doi:10.1029/2002JD002363, 2003.

Vadas, S.-L. and Fritts, D.-C.: Gravity wave radiation and mean responses to local body forces in the atmosphere, J. Atmos. Sci., 58, 2249-2279, 2001. 